ORIGINAL ARTICLE

\title{
Prevalence of Pneumonia Associated with Measles among Infants and Children
}

\author{
ASADULLAH KHAN ${ }^{1}$, ABDUL MOEED KHAN ${ }^{2}$, ALI AKBAR $^{3}$, MUHAMMAD AKRAM $^{4}$, FARHANA AHMAD $^{5}$, ALLAH NAWAZ $^{2}$ \\ SULTAN ${ }^{6}$ \\ ${ }^{1}$ Specialist Paediatrician Alkhidmat Hospital, Peshawar \\ ${ }^{2}$ FCPS Paediatrics, Fellow Paediatric Cardiology Lady Reading Hospital, Peshawar \\ ${ }^{3}$ FCPS Pediatrics, Fellow Pediatric Cardiology Lady Reading Hospital, Peshawar \\ ${ }^{4}$ Assistant Professor Pediatrics, Sharif Medical and Dental College Lahore \\ ${ }^{5}$ Assistant Professor Pediatrics, Central Park Medical College, Lahore \\ ${ }^{6}$ Assistant Professor Pediatrics, Sharif Medical and Dental College, Lahore \\ Corresponding author: Dr. Ali Akbar, Email: drakbarpeds01018@gmail.com. Cell No.03219109226
}

\begin{abstract}
Objective: The aim of this study is to determine the prevalence of pneumonia associated with measles among children and infants.

Study Design: Cross-sectional

Place and Duration: Pediatrics department of Lady Reading Hospital, Peshawar and Sharif Medical and Dental College, Lahore for six months duration from February 2020 to July 2020.

Methods: Total 150 patients i.e children and infants of both genders were included in this study. Children were aged <5years of age. Patients' detailed demographics height and weight were recorded after taking informed written consent from the authorities. Patients' diagnosed measles were presented. Symptoms and frequency of pneumonia among all patients were assessed. Complete data was analyzed by SPSS 22.0 version.

Results: There were 90 (60\%) children and 60 (40\%) infants. Among 150 patients, $100(66.7 \%)$ were males and $50(33.3 \%)$ were females. $70(46.7 \%)$ cases had birth weight $>3 \mathrm{~kg} .30(20 \%)$ patients had high socioeconomic status, 50 (33.3\%) had middle and 70 (46.6\%) cases had poor. $85(56.7 \%)$ patients had rural residency. According to severity of measles, $35(23.3 \%)$ had mild,45 (30\%) cases had moderate and severity was among 70 (46.7\%) patients. Prevalence of pneumonia was found among 95 (63.3\%) cases. 40 (26.75) cases had measles vaccination.

Conclusion: We concluded in this study that prevalence of pneumonia among children of measles were significantly high. It was due to less number of vaccination status with poor socio economic status among patients. It can be controlled by providing awareness of vaccination to the parents of children.
\end{abstract}

Keywords: Pneumonia, Measles, Children, Infants, Prevalence

\section{INTRODUCTION}

Infected individuals spread the disease by inhaling droplets or aerosols from the respiratory fluids of infected individuals [1]. Measles has an incubation period of 7-21 days. Fevers and generalized maculopapular erythematous patches, cough, coryza and conjunctivitis are symptoms of measles infection. Pneumonia (1/20 occurrences), otitis media (1/10 cases), thrombocytopenia, diarrhea (1/10 cases), and encephalitis might arise up to several weeks following the rash (1:1000 cases). It is possible that measles can cause blindness, deafness, intellectual disabilities associated with encephalitis (acute disseminated encephalomyelitis in approximately 1:000 cases; subacute sclerosing panencephalitis in approximately $1: 10,000$ to $1: 100,000$ cases), and death (1:3000) in malnourished children [1]. Every year, the number of measles cases that die ranges from less than one in 1000 to 5 percent for endemic areas of sub-Saharan Africa and Asia, to as high as 20-30 percent for refugees and internally displaced people [1].

As a MIC in Europe, Romania has recommended two doses of measles-containing vaccination (MCV) for children since 1994. Since 2002, the first dose (MCV1) is generally given at 12 months of age, and the second dose (MCV2) is normally given at 5 years of age [2. During the period 20002010 , Romania had an MCV1 coverage rate of $>95 \%$. After the MCV1 coverage in Romania began to decline in 2011, it dropped to about 86 percent per year from 2015 to
2017 [3] National MCV coverage declined as a result of a vociferous and powerful anti-vaccine movement as well as issues within the immunization infrastructure relating to the availability of vaccines for routine immunization and vaccine delivery [4].

Measles outbreaks continue in Romania, despite WHO European Region's goal of eliminating measles [5], with 8709 cases reported from 2004 to 2007 and 12,991 cases reported from 2010 to 2013 [6]. On July 27, 2018, Romania's Ministry of Health (MOH) confirmed 14,825 measles cases. The most current outbreak began in 2016 . 24,5 percent of the total 14,825 cases were reported in 2016, 9076 percent were recorded in 2017, and 3314 (22.5 percent) have been reported till July 2018. In May of 2017, the outbreak looked to have peaked. However, in May of 2018, another surge was recorded. Fourteen hundred and twenty-five of of the fourteen thousand and twenty-five measles cases were unvaccinated, with 8259 (56 percent) of the cases occurring in infants and young children [6].

A patient's severity and complication of measles and measles-associated pneumonitis will determine their treatment. To control and reduce the severity of pneumonia and measles complications, mostly supportive therapy is required [7]. Otitis media and bacterial pneumonia are the most common consequences. It is necessary to admit patients with serious problems, such as encephalomyelitis, for supervision during the period when airborne 
transmission occurs in admitted children. Children who die from measles are given vitamin $A$ by the World Health Organization.[8] Depending on their age, the dose should be given. In typically, the measles vaccine is given along with the Mumps and Rubella vaccines (MMR). If administered within three days of exposure, the vaccination is protective.

\section{MATERIAL AND METHODS}

This cross sectional study was conducted at Pediatrics department of Lady Reading Hospital, Peshawar and Sharif Medical and Dental College, Lahore for six months duration from February 2020 to July 2020. and comprised of 150 patients. Patients' detailed demographics were recorded after taking informed written consent. Patients greater than 5 years of age and had other medical illness were excluded from this study.

Total 150 patient's i.e children and infants of both genders were included in this study. Children were aged $<5 y e a r s$ of age. Patients' detailed demographics height and weight were recorded after taking informed written consent from the authorities. Patients diagnosed measles were presented. Symptoms and frequency of pneumonia among all patients were assessed. Complete data was analyzed by SPSS 22.0 version. Categorical variables were assessed by frequencies and percentages.

\section{RESULTS}

There were 90 (60\%) children and 60 (40\%) infants. Among 150 patients, $100(66.7 \%)$ were males and 50 $(33.3 \%)$ were females. $70(46.7 \%)$ cases had birth weight $>$ $3 \mathrm{~kg} .30(20 \%)$ patients had high socioeconomic status, 50 (33.3\%) had middle and $70(46.6 \%)$ cases had poor. 85 $(56.7 \%)$ patients had rural residency. (Table 1 )

Table 1: Baseline details demographics of enrolled cases

\begin{tabular}{|l|l|l|}
\hline Characteristics & Frequency & $\%$ age \\
\hline Children & 90 & 60 \\
\hline Infants & 60 & 40 \\
\hline Gender & 100 & 66.7 \\
\hline Male & 50 & 33.3 \\
\hline Female & 70 & 46.7 \\
\hline Birth Weight >3kg & 80 & 53.3 \\
\hline Yes & \multicolumn{1}{|l|}{} \\
\hline No & 70 & 46.7 \\
\hline Socio economic status & 33.3 \\
\hline Poor & 50 & 20 \\
\hline Middle & 30 & 56.7 \\
\hline High & 85 & 43.3 \\
\hline Residency & 65 & \\
\hline Rural & \multicolumn{1}{|l|}{} \\
\hline Urban &
\end{tabular}

According to severity of measles, 35 (23.3\%) had mild, $45(30 \%)$ cases had moderate and severity was among 70 (46.7\%) patients.(table 2)

Table 2: Association of measles with respect to severity

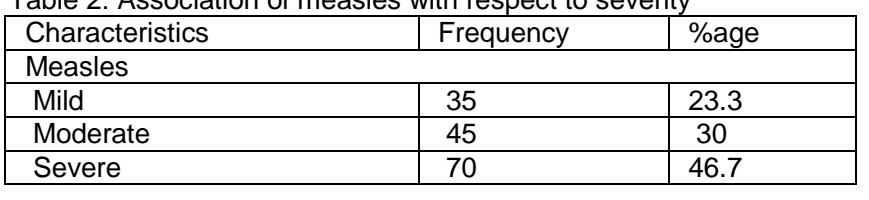

Prevalence of pneumonia was found among 95 $(63.3 \%)$ cases. $40(26.75)$ cases had done measles vaccination.(table 3 )

Table 3: Prevalence of pneumonia and vaccination status among all patients

\begin{tabular}{|l|l|l|}
\hline Characteristics & Frequency & \%age \\
\hline Pneumonia & 95 & 63.3 \\
\hline Yes & 55 & 36.7 \\
\hline No & 40 & 26.75 \\
\hline Vaccination Status & 110 & 73.25 \\
\hline Yes
\end{tabular}

\section{DISCUSSION}

Lung infection caused by the measles posed a statistically significant risk for death. Measles can be a "second punch" for previously sick children and newborns with acute respiratory illnesses such as influenza, according to a study from Mongolia [9]. Neurologic sequelae, such as encephalitis and seizures, were also indicators of infection severity in both cases and controls. This unusual consequence only affects about $0.1 \%$ of measles cases [10]. An inflammatory process is believed to be the primary cause of postinfectious myelitis and/or encephalitis (PIE), which occurs after measles infection, usually within 2 weeks after the rash [10].

In this cross-sectional study 150 patients of both genders were enrolled. Among 150 patients, 90 (60\%) were children and the rest $60(40 \%)$ were infants. Majority of the patients $66.7 \%$ were males. Our findings were comparable to the previous study.[11] 70 (46.7\%) cases had birth weight $>3 \mathrm{~kg} .30(20 \%)$ patients had high socioeconomic status, $50(33.3 \%)$ had middle and 70 $(46.6 \%)$ cases had poor. 85 (56.7\%) patients had rural residency.[12] Similar results were also presented by other authors.[13]

In our study 35 (23.3\%) had mild,45 (30\%) cases had moderate and severity of measles was among 70 (46.7\%) patients. [14] Prevalence of pneumonia was found among $95(63.3 \%)$ cases. This was high children and infants. A research in Peshawar found that pneumonia was the most common consequence among patients, accounting for 68 percent [15]. According to a Swedish study, 75\% of infants under one year old had pneumonia. Pneumonia affects 40 percent more males than girls [16]. In our study $26.8 \%$ cases received vaccination of measles. In children with measles, the $\mathrm{WHO}$ recommends giving vitamin $\mathrm{A}$ once a day for two days in a row $(50,000$ international units (IU) for infants younger than six months, $100,000 \mathrm{IU}$ for infants aged 6-11 months, and 200,000 IU for children more than one year old) [17]. Lower measles-specific antibody levels and greater morbidity may be linked to vitamin A deficiency Vitamin A insufficiency in a malnourished youngster can result in a lack of hepatic vitamin $A$ reserves when infected with measles.[18]

All hospitals should employ infection prevention and control procedures to avoid nosocomial measles virus spread. Patients with known or suspected measles should be quickly identified and isolated, and health care staff should follow conventional and airborne precautions [19]. Measles deaths are "preventable tragedies that may have been averted via vaccination" [20] because there is a safe 
and effective measles vaccine. Pneumonia is more common in the current study than in earlier investigations. A high immunization rate may be the most likely cause of the problem. Undernutrition is also a risk factor for death due to pneumonitis in spite of $60 \%$ of children being seriously afflicted [21].

\section{CONCLUSION}

We concluded in this study that prevalence of pneumonia among children of measles were significantly high. It was due to less number of vaccination status with poor socio economic status among patients. It can be controlled by providing awareness of vaccination to the parents of children.

\section{REFERENCES}

1. Moss WJ. Measles. Lancet (London, England). 2017;390(10111):2490-502.

2. World Health Organization. Immunization, vaccines and biologicals: data, statistics and graphics. Available from: https://www.who.int/immunization/monitoring surveilla nce/data/en/. Accessed 30 Apr 2020.

3. World Health Organisation. Romania: WHO and UNICEF Estimates of National Immunization Coverage (WUENIC): 2018 revision [Available from: https://www.who.int/immunization/monitoring_surveilla nce/data/rou.pdf]. Accessed 30 Apr 2020

4. Zimmerman LA, Muscat $M$, Singh $S$, Ben Mamou $M$, Jankovic D, Datta S, et al. Progress toward measles elimination - European region, 2009-2018. MMWR Morb Mortal Wkly RepMMWR. 2019;68(17):396-401.

5. World Health Organization. Reported cases of selected vaccine-preventable diseases. Available from: https://www.who.int/immunization/monitoring_surveilla nce/data/en/. Accessed 7 May 2020.

6. Romania National Public Health Institute (INSP). Romania measles surveillance data. Available from: http://www.cnscbt.ro/index.php/informarisaptamanale/rujeola-1/. Accessed 30 Apr 2020.

7. Narain JP \& Banerjee KB (1989). Measles in India Epidemiology and control. Indian J Pediatr 56: 463-472.

8. Pongrithsukda VIM, Olmarn, Phonboon Kanchanasak \& Manunpichu, Krittaya (1986). Measles-associated diarrhoea in northeastern Thailand. Se Asian J Trop Med 17(1): 43.

9. Lee CT, Hagan JE, Jantsansengee B, Tumurbaatar OE, Altanchimeg S, Yadamsuren B, et al. Increase in infant measles deaths during a Nationwide measles outbreak Mongolia, 2015-2016. J Infect Dis. 2019;220(11):1771-9.
10. Rafat C, Klouche K, Ricard JD, Messika J, Roch A, Machado $\mathrm{S}$, et al. Severe measles infection: the Spectrum of disease in 36 critically ill adult patients. Medicine. 2013;92(5):25772.

11. Shahid Khan, Jafar Iqbal, Muhammad Tayyeb, Shah Fahad, Amen Ullah and Hidayat Khan. Prevalence of pneumonia associated with measles among infants and children hospitalized in Khyber Teaching Hospital Peshawar, KPK, Pakistan. Pure and Applied Biology.Vol. 10, Issue 3, pp703712

12. Jayashree Gothankar, Prakash Doke , Girish Dhumale Prasad Pore , Sanjay Lalwani , Sanjay Quraishi , Sujata Murarkar, Reshma Patil , Vivek Waghachavare, Randhir Dhobale, Kirti Rasote, Sonali Palkar and Nandini Malshe. Reported incidence and risk factors of childhood pneumonia in India: a community-based cross-sectional study. BMC Public Health (2018) 18:1111

13. Ahsan MR, Al Mamun A, Alam HSK, Sarker PK, Makbul S, Kabir R \& Al Mamun AMH (2018). Occurrence of Measles among Children Admitted in Tertiary Care Hospital. Bangladesh J. Child Health 42(1): 15-18.

14. Sato M, Tateishi, R, Yasunaga $\mathrm{H}$, Horiguchi $\mathrm{H}$, Yoshida $\mathrm{H}$ Matsuda S \& Koike, K (2012). Mortality and morbidity of hepatectomy, radiofrequency ablation and embolization For Hepato cellular carcinoma: a national survey of 54,145 patients. J Gastroenterol Hepatol 47(10): 1125-1133.

15. Ogbuanu IU, Zeko S \& Chu SY (2014). Maternal, fetal, and neonatal outcomes associated with measles during pregnancy: Namibia, 2009-2010. Clin Infect Dis 58: 1086.

16. Berglund A, Ekelund M, Fletcher MA \& Nyman L (2014). Allcause pneumonia hospitalizations in children $<2$ years old in Sweden, 1998 to 2012: impact of pneumococcal conjugate vaccine introduction. PLoS One 9(11): e112211

17. World Health Organization. Guide for clinical case management and infection prevention and control during a measles outbreak. [updated 2020. Available from: https://apps.who.int/iris/bitstream/handle/10665/33159 9/9789240002869-eng.pdf?ua=1.

18. Burke RM, Whitehead RD Jr., Figueroa J, Whelan D, Aceituno AM, Rebolledo PA, Revollo R, Leon JS, Suchdev PS. Effects of Inflammation on Biomarkers of Vitamin A Status among a Cohort of Bolivian Infants. Nutrients. 2018;10(9):1240.

19. Maltezou HC, Wicker S. Measles in health-care settings. Am J Infect Contr. 2013;41(7):661-3.

20. Paules $\mathrm{Cl}$, Marston HD, Fauci AS. Measles in 2019 - going backward. N Engl J Med. 2019;380(23):2185-7.

21. Donadel, M., Stanescu, A., Pistol, A. et al. Risk factors for measles deaths among children during a Nationwide measles outbreak - Romania, 2016-2018. BMC Infect Dis 21,279 (2021). 